\title{
Mediastinal Anaplastic Ependymoma
}

\author{
Dyah Fauziah, M.D. ', Irene Lingkan Parengkuan, M.D. ${ }^{1,2}$, Dhihintia Jiwangga, M.D. ${ }^{3}$, Paulus Raharjo, M.D. ${ }^{4}$, \\ Mudjiani Basuki, M.D. ${ }^{5}$
}

${ }^{1}$ Department of Anatomical Pathology, Dr. Soetomo Hospital, Faculty of Medicine, Universitas Airlangga; ${ }^{2}$ Department of Anatomical Pathology, Faculty of Medicine, Universitas Katolik Widya Mandala; Departments of ${ }^{3}$ Thoracic, Cardiac and Vascular Surgery, ${ }^{4}$ Radiology, and ${ }^{5}$ Neurology, Dr. Soetomo Hospital, Faculty of Medicine, Universitas Airlangga, Surabaya, Indonesia

\section{ARTICLE INFO}

Received July 13, 2020

Revised September 22, 2020

Accepted October 12, 2020

Corresponding author

Dyah Fauziah

Tel 62-31-5020251 (ext: 151 and 153)

Fax 62-31-5022472

E-mail dyahf73@gmail.com;

dyah-f@fk.unair.ac.id

ORCID

https://orcid.org/0000-0002-7661-1647
Ependymomas arise from ependymal cells and can grow at any site in the central nervous system (CNS), as well as in some locations outside of the CNS. The latter is rare, contributing to the frequent misdiagnoses of such cases. Herein, we present the case of a 54-year-old man with a history of lower limb weakness and numbness. Magnetic resonance imaging revealed an extradural, heterogeneously enhanced solid lesion with a regular and well-defined border in the posterior mediastinum. A post-resection histopathological examination revealed tumor-forming perivascular pseudo-rosettes that showed immunoreactivity against glial fibrillary acidic protein, epithelial membrane antigen, and vimentin, as well as a high Ki-67 labeling index. Based on pathological features, a diagnosis of anaplastic ependymoma was established.

Keywords: Mediastinum, Neurogenic tumor, Ependymoma

\section{Case report}

A 54-year-old man was referred to the neurology outpatient clinic of Dr. Soetomo Hospital, Surabaya, Indonesia. His main complaint was weakness of both lower limbs in the last 4 months, followed by numbness, urinary incontinence, and constipation. He also complained of pain in his spine that had lasted for 5 months. He reported no injuries, and his medical history was not significant.

A physical examination found a wound with pus in the lumbar area. The patient had normal vital signs. A neurological examination found 5/0 upper and lower muscle strength, with positive physiological reflexes and negative findings for pathological reflexes. The findings of routine laboratory examinations were unremarkable.

Magnetic resonance imaging (MRI) of the thoracolumbar region found a solid lesion with a well-circumscribed and regular border in the posterior mediastinum area, measuring $7.5 \times 5.3 \times 9 \mathrm{~cm}$, with an extradural location on the left side of the ninth thoracic vertebra, expanding to the medial side and compressing the spinal cord. The tumor showed contrast enhancement (Fig. 1).

The patient underwent tumor resection through a left

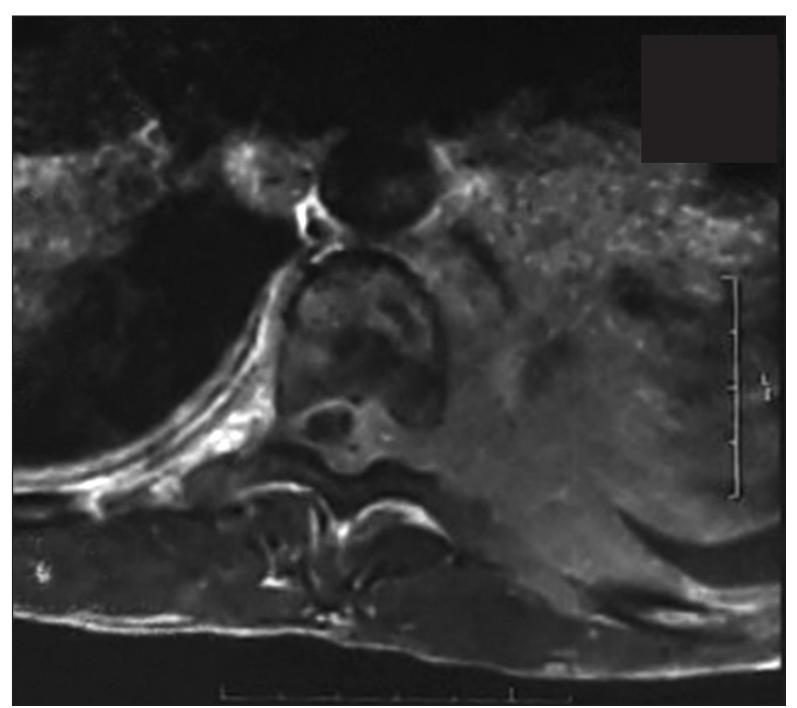

Fig. 1. Magnetic resonance imaging showed a tumor in the posterior mediastinum.

posterolateral thoracotomy. The tumor was very large, located close to the aorta, and infiltrated the vertebra. The tumor was removed completely without serious morbidity.

The resection specimen showed a solid tumor with a soft 

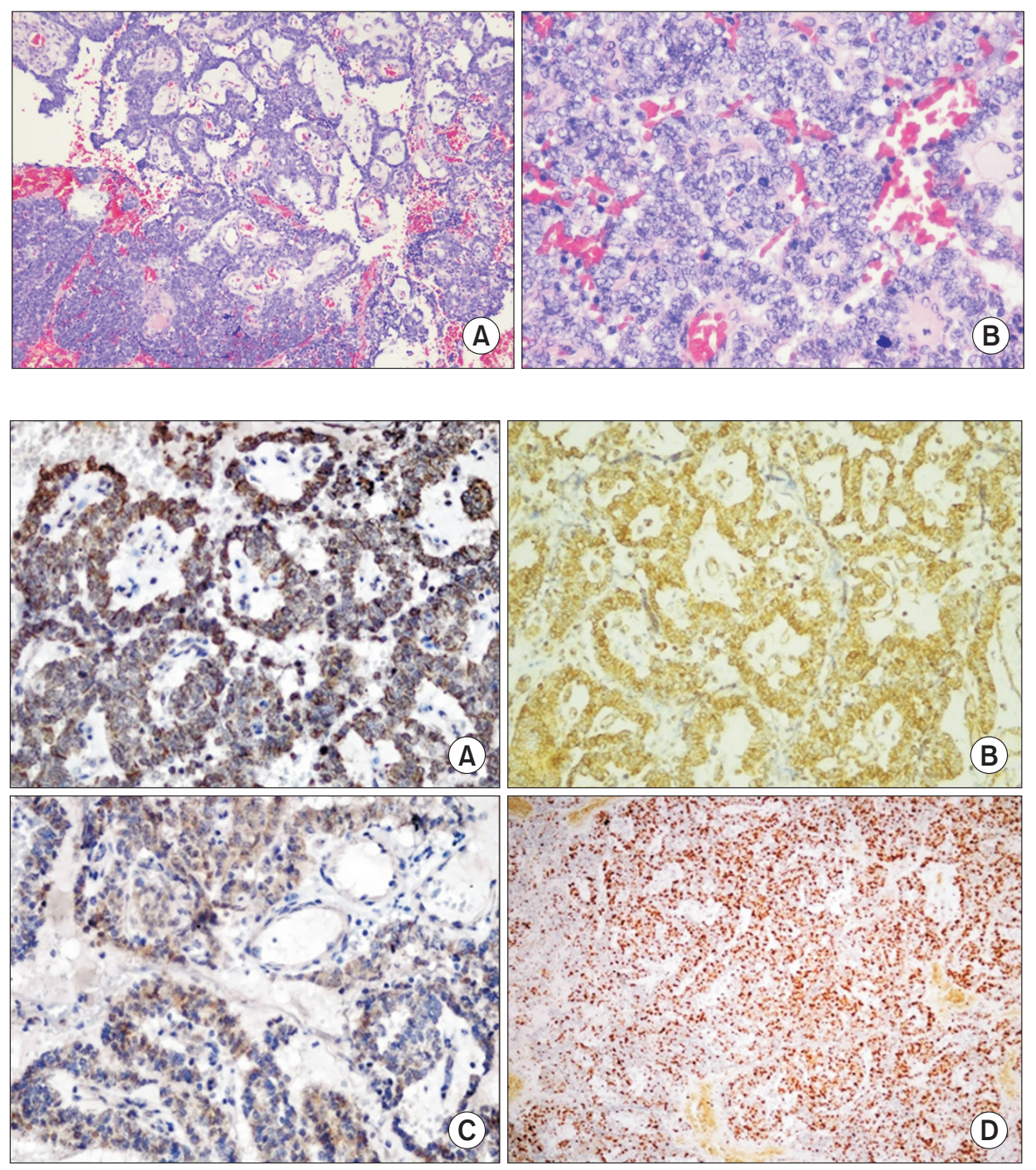

Fig. 2. (A) Microscopic features showed perivascular pseudo-rosettes in the central and upper part, and solid areas in the left and lower part $(\mathrm{H} \& \mathrm{E}, \times 100)$. (B) Solid area of the tumor with mitotic activity (H\&E, $\times 400)$.

Fig. 3. (A-C) Tumor cells were positive for glial fibrillary acidic protein, vimentin, and epithelial membrane antigen, respectively $(\times 200, \times 200$, $\times 400$ ). (D) The tumor showed a high Ki-67 labeling index $(\times 40)$. consistency and a grayish-white to tan color, with a total weight of $163 \mathrm{~g}$ and dimensions of $10.5 \times 6 \times 4.5 \mathrm{~cm}$. Microscopically, the tumor was arranged in a solid and pseudopapillary pattern, and perivascular pseudo-rosettes were observed (Fig. 2A). The tumor cells were cuboid-shaped with rounded nuclei, mild pleomorphism, and coarse chromatin. The mitotic count in the solid area of the tumor was high (Fig. 2B). An immunohistochemical examination showed that the tumor cells were positive for vimentin, glial fibrillary acidic protein (GFAP), and epithelial membrane antigen (EMA) (Fig. 3A-C) and showed a high Ki-67 labeling index (Fig. 3D). Based on these findings, the tumor was diagnosed as anaplastic ependymoma, consistent with World Health Organization grade 3.

This study received ethical approval from the Institutional Review Board of Dr. Soetomo Hospital, Surabaya, East Java, Indonesia, with the following number: reference no., 0170/LOE/301.4.2/X/2020.

\section{Discussion}

Ependymoma is a neoplasm arising from ependymal cells. Ependymoma can occur in all ages, but has 2 main peaks (around 0-4 years old and 55-59 years old). It can grow along the central nervous system (CNS) compartment or even outside of the CNS $[1,2]$.

Ependymomas that primarily occur outside of the CNS are rarely found. Extra-CNS ependymoma can occur in the sacrococcygeal, pelvic, and extra-pelvic areas, of which the sacrococcygeal area is the most commonly reported location. Pelvic ependymoma can occur in the ovary and its supporting tissue. Ependymoma in the ovary can show the histopathological features of a true ependymoma or appear as part of a teratoma [3]. Zhou et al. [4] reported a case of 
pelvic ependymoma in the lateral pelvic wall that adhered to gastrointestinal organs. Due to its rarity and variable morphological patterns, pelvic ependymoma may initially be misdiagnosed as another malignant tumor. The most common location of extra-pelvic ependymoma is the mediastinum [5].

Herein, we report a case of ependymoma in the posterior mediastinum with an extradural location separate from the CNS. The posterior mediastinum is a common location for the growth of neurogenic neoplasms such as neurofibroma and schwannoma in adults. The imaging features of those 2 types of tumors include homogeneous or heterogeneous high signal intensity on T2-weighted images. Cystic changes and hemorrhages are more common in schwannomas than in neurofibromas. Primary mediastinal ependymoma is rarely found in this location, and usually appears as a well-defined mass with variable enhancement on computed tomography or MRI imaging.

The etiology of primary extra-CNS ependymoma has not yet been clarified. Sacrococcygeal ependymoma is known to derive from the remaining ependymal cells that are trapped as a result of the regression process and the physiological collapse of the central canal, while the pathogenesis of pelvic and extra-pelvic ependymoma is thought to be related to disturbances in the migration of primordial germ cells. The latter arise from yolk sac endoderm and migrate through the cloaca, hindgut, and dorsal mesentery towards the gonadal ridge, where the ovary and its connective tissue grows. Ependymomas that grow in the ovary, broad ligament, and mediastinum are likely to be derived from misdirected primordial germ cells [5].

Extra-CNS ependymoma is often initially misdiagnosed due to its rarity. The characteristic features of perivascular pseudo-rosettes should alert clinicians to the diagnosis. Anaplastic ependymoma is established when ependymoma shows an increase of cellular density and high mitotic activity with microvascular proliferation and necrosis [2]. The histopathologic features in this case were consistent with anaplastic ependymoma.

The differential diagnosis based on morphology includes neuroblastoma and primitive neuroectodermal tumor (PNET). These tumors show monotonous blue round cells with rosette formation. Neuroblastoma and PNET both show the characteristic feature of Homer Wright rosettes. In this case, we observed perivascular pseudo-rosettes, which are typically found in ependymomas [6]. The clinical context is also important for the diagnosis, as neuroblastoma and PNET mostly affect children or young adults [7].
Immunohistochemistry is required for establishing a diagnosis in difficult cases. Anaplastic ependymoma shows immunoreactivity towards GFAP, S-100, vimentin, and EMA, as well as a high Ki-67 labeling index [2]. The correlations between clinical, radiological, and pathological findings are important for diagnosing rare tumors, such as extra-CNS ependymoma [3-5].

Although ependymoma is rarely found in the mediastinum, the present case may raise clinicians' awareness of this tumor. Diagnosis of this tumor is important due to its malignant behavior, unlike other common neurologic tumors such as schwannoma or neurofibroma.

\section{Conflict of interest}

No potential conflict of interest relevant to this article was reported.

\section{ORCID}

Dyah Fauziah: https://orcid.org/0000-0002-7661-1647 Irene Lingkan Parengkuan: https://orcid.org/0000-0002-7407-9052 Dhihintia Jiwangga: https://orcid.org/0000-0002-6767-0768 Paulus Raharjo: https://orcid.org/0000-0002-1821-4811

Mudjiani Basuki: https://orcid.org/0000-0002-5370-7750

\section{References}

1. Villano JL, Parker CK, Dolecek TA. Descriptive epidemiology of ependymal tumours in the United States. Br J Cancer 2013;108: 2367-71.

2. Elison DW, McLendon R, Wiestler OD, et al. Ependymal tumours. In: Louis DN, Ohgaki H, Wiestler OD, Cavenee WK, editors. WHO classification of tumours of the central nervous system. 4th ed. Lyon: International Agency for Research on Cancer; 2016. p. 101-14.

3. Kleinman GM, Young RH, Scully RE. Ependymoma of the ovary: report of three cases. Hum Pathol 1984;15:632-8.

4. Zhou F, Song J, Mikolaenko I, Rosenblum M, Shukla PS. Pelvic ependymoma with clinical response to GnRH analog therapy: a case report with an overview of primary extraneural ependymomas. Int $\mathrm{J}$ Gynecol Pathol 2015;34:450-8.

5. Maeda S, Takahashi S, Koike K, Sato M. Primary ependymoma in the posterior mediastinum. Ann Thorac Cardiovasc Surg 2011;17: 494-7.

6. Ahmed MU. Rosettes and pseudorosettes and their significance. J Enam Med Coll 2017;7:101-6.

7. Rajwanshi A, Srinivas R, Upasana G. Malignant small round cell tumors. J Cytol 2009;26:1-10. 